\title{
ARTIFICIAL BEE COLONY CONSTRAINED OPTIMIZATION ALGORITHM WITH HYBRID DISCRETE VARIABLES AND ITS APPLICATION
}

\author{
Zhonghua Yan \\ Changde Textile Machinery Co. Ltd, Hunan, Changde, 415001, P.R.China \\ *Corresponding Author Email: 13873670621@163.com
}

This is an open access article distributed under the Creative Commons Attribution License, which permits unrestricted use, distribution, and reproduction in any medium, provided the original work is properly cited.

\section{ARTICLE DETAILS}

Article History:

Received 12 November 2017 Accepted 12 December 2017

Available online 1 January 2018

\section{ABSTRACT}

On the basis of Artificial Bee Colony Optimization Algorithm, a new algorithm by introducing constructing dynamic penalty function was presented. Based on Matlab software, the program ABCOA1.0 with hybrid discrete variables for the proposed algorithm was developed. The results show that this algorithm has no special requirements on the characteristics of optimal designing problems, which has a fairly good universal adaptability and a reliable operation of program with a strong ability of overall convergence. After optimization, the weight can be reduced, the cost can be lowered, and the product quality can be raised.

\section{KEYWORDS}

Artificial Bee Colony Optimization Algorithm(ABCOA), Constrained optimization, hybrid discrete variable, Dynamic Penalty Function, Evolutionary Algorithm.

\section{INTRODUCTION}

Optimization design is a very effective way to ensure that the product has excellent performance, lighten weight and volume and reduce the product cost, and it has been widely used in product design [1-6]. The optimization problem is to find multiple optimal solutions of multi-modal problems in the feasible region, thus it provides multiple choice or multi-faceted information for decision makers [7]. It is the optimization design problem with hybrid discrete variables containing integer variables, discrete variables and continuous variables for the engineering optimization design. It is the most meaningful but more difficult in mathematical programming and operations research for discrete variable optimization $[8,9]$. Many classical numerical methods for optimizing problem can actually get good results for some problems. But these methods such as continuous and differentiable have strong constraint for the objective function, and they have strong dependence on optimization problem. At the same time, the algorithm results are related to the selection of initial values and easily trapped in local minimum. When dealing with complex multimodal optimization problem, the traditional search method for single point often only searches uncertainty one extreme point one time, so it is basically invalid for the complicated multimodal optimization problem. The developing swarm intelligence algorithms become new effective approaches for solving multi-peak function optimization. In recent years, the booming evolutionary algorithm with the global optimality, parallelism and efficiency has been widely used in function optimization. With the help of the evolution of nature, the evolutionary algorithm overcoming the drawback of traditional numerical method as a global optimization method for multiple clues is based on the population and random search mechanism. It has been attracted widespread attention of evolutionary computation in the field of optimization application, and various forms of evolution algorithm emerge in endlessly. Colonies of social insects such as ants and bees have instinct ability known as swarm intelligence $[10,11]$. This highly organized behavior enables the colonies of insects to solve problems beyond capability of individual members by functioning collectively and interacting primitively amongst members of the group. In a honey bee colony for example, this behavior allows honey bees to explore the environment in search of flower patches (food sources) and then indicate the food source to the other bees of the colony when they return to the hive. Such a colony is characterized by selforganization, adaptive and robustness. On the basis of Artificial Bee Colony Optimization Algorithm, an new algorithm by introducing constructing dynamic penalty function was presented [12]. Based on Matlab software, the program ABCOA1.0 with hybrid discrete variables for the proposed algorithm was developed. The example shows that this algorithm had no special requirements on the characteristics of optimal designing problems, which has a fairly good universal adaptability and a reliable operation of program with a strong ability of global convergence.

\section{ARTIFICIAL BEE COLONY CONSTRAINED OPTIMIZATION ALGORITHM WITH HYBRID DISCRETE VARIABLES}

\subsection{Artificial Bee Colony Optimization Algorithm}

In a real bee colony, there are some tasks performed by specialized individuals. These specialized bees try to maximize the nectar amount stored in the hive by performing efficient division of labour and selforganization. The minimal model of swarm-intelligent forage selection in a honey bee colony, that $\mathrm{ABC}$ algorithm adopts, consists of three kinds of bees: employed bees, onlooker bees, and scout bees. Half of the colony comprises employed bees and the other half includes the onlooker bees. Employed bees are responsible from exploiting the nectar sources explored before and giving information to the other waiting bees (onlooker bees) in the hive about the quality of the food source site which they are exploiting. Onlooker bees wait in the hive and decide a food source to exploit depending on the information shared by the employed bees. Scouts randomly search the environment in order to find a new food source depending on an internal motivation or possible external clues or randomly. Main steps of the $\mathrm{ABC}$ algorithm are given as follow:

Step 1: Initialize the food source positions

Step 2: Each employed bee produces a new food source in her food source site and exploits in the better source

Step 3: Each onlooker bee selects a source depending on the quality of her solution, produces a new food source in selected food source site and exploits the better source.

Step 4: Determine the source to be abandoned and allocate its employed bee as scout for searching new food sources.

Step 5: Memorize the best food source found so far.

Step 6: Repeat steps 2-5 until the stopping criterion is met.

The above method is used for unconstrained optimization problems $[10,11]$.

Considering that unconstrained problems have lots of solving methods, it is a natural thought to transform the constrained problem to 
unconstrained problem. The most common way is to use penalty function. Construct a kind of penalty function according to the feature of restraints and then add it to the objective function. In the solving process of unconstrained problem, this penalty strategy can assign a large objective function value to the iteration points which are against the restraints (this is a penalty to the minimization), and then a series of minimum points in unconstrained problem will infinitely close to or moving in the feasible region, till the iteration points converge to the minimum points of the former constrained problem.

The transformed unconstrained problem can be described as follows [12].

$$
G(\mathbf{x})=F(\mathbf{x})+H(\mathbf{x}) h(t)
$$

Where, $F(\mathbf{x})$ is the fitness function, $h(t)$ is the penalty degree function, $t$ is the evolvable generations, $H(\mathbf{x})$ is the penalty factor and be defined as

$$
H(\mathbf{x})=\sum_{i=1}^{m} \mu\left(\phi_{i}(\mathbf{x})\right) \phi_{i}(\mathbf{x})^{\delta\left(\phi_{i}(\mathbf{x})\right)}
$$

Where, $\phi_{i}(\mathbf{x})=\max \left\{0, g_{i}(\mathbf{x})\right\}, g_{i}(\mathbf{x})$ is constrained function, functions $h(\bullet), \quad \mu(\bullet), \quad \delta(\bullet)$ depend on the specific problem.

\subsection{Engineering process method of design variable}

\subsubsection{The discretization of discrete variable}

In Artificial Bee Colony Optimization Algorithm, the new bee colony after updating is continuous and must be carried on discretization. The process of integer variables is similar to the mentioned discretization of nonequidistant discrete variable, the only difference is that the value range is nonnegative integers in the specific threshold [12]

\subsubsection{Engineering process of continuous variable}

In optimum designing, the values of some variables are limited by the mechanical accuracy and design specification although they are theoretically continuous. If calculate them with floating-point real number or double-precision real number but process the final data with the decimal required by practice, then the final design scheme is not the optimum solution or doesn't satisfy the constrained condition. Thus, the decimal accuracy in calculation should meet the practical requests [1214].

\subsection{Compiling program}

Based on Matlab software, the program ABCOA1.0 of Artificial Bee Colony Optimization Algorithm with hybrid discrete variables for the proposed algorithm was developed with Matlab.

\section{OPTIMIZATION CASE STUDY}

The optimum model of one single gear reducer [13]

$$
\begin{aligned}
& \min \quad f(\mathbf{x})=0.78539815\left(4.75 x_{1} x_{2}^{2} x_{3}^{2}+85 x_{1} x_{2} x_{3}^{2}-85 x_{1} x_{3}^{2}+0.92 x_{1} x_{6}^{2}-x_{1} x_{5}^{2}+0.8 x_{1} x_{2} x_{3} x_{6}\right. \\
& \left.-1.6 x_{1} x_{3} x_{6}+x_{4} x_{5}^{2}+x_{4} x_{6}^{2}+32 x_{6}^{2}+28 x_{5}^{2}\right) \\
& \text { S.T. } \quad g_{1}(\mathbf{x})=17-x_{2} \leq 0 \\
& g_{2}(\mathbf{x})=30-x_{2} X_{3} \leq 0 \\
& g_{3}(\mathbf{x})=0.2-x_{3} \leq 0 \\
& g_{4}(\mathbf{x})=16-x_{1} / x_{3} \leq 0 \\
& g_{5}(\mathbf{x})=x_{1} / x_{3}-35 \leq 0 \\
& g_{6}(\mathbf{x})=10-x_{5} \leq 0 \\
& g_{7}(\mathbf{x})=x_{5}-15 \leq 0, g_{8}(\mathbf{x})=13-x_{6} \leq 0 \\
& g_{9}(\mathbf{x})=x 6-20 \leq 0 \\
& g_{10}(\mathbf{x})=x_{1}+0.5 x_{6}-x_{4}+4 \leq 0 \\
& g_{11}(\mathbf{x})=\frac{43854}{x_{2} X_{3} \sqrt{X_{1}}}-855 \leq 0 \\
& g_{12}(\mathbf{x})=\frac{7098}{X_{1} X_{2} X_{3}^{2} W_{1}}-261 \leq 0 \\
& g_{13}(\mathbf{x})=\frac{7098}{X_{1} X_{2} X_{3}^{2} W_{2}}-213 \leq 0 \\
& g_{14}(\mathbf{x})=\frac{0.01233 x_{4}^{3}}{x_{1} X_{3} x_{5}^{4}}-0.003 x_{4}-261 \leq 0 \\
& g_{15}(\mathbf{x})=29050 \frac{29050 x_{4}}{x_{2} x_{3} x_{5}^{3}} \sqrt{1+\frac{0.29709 x_{2}^{2} x_{3}^{2}}{x_{4}^{2}}}-55 \leq 0 \\
& g_{16}(\mathbf{x})=\frac{29050 x_{4}}{x_{2} X_{3} x_{6}^{3}} \sqrt{1+\frac{7.42727 x_{2}^{2} X_{3}^{2}}{x_{4}^{2}}}-55 \leq 0 \\
& W_{1}=\left(0.169+0.006666 x_{2}-0.0000854 x_{2}^{2}\right. \\
& W_{2}=0.2824+0.00177 x_{2}-0.0000394 x_{2}^{2}
\end{aligned}
$$

Where, the objective function $f(\mathbf{x})$ is the volume of reducer $\left(\mathrm{cm}^{3}\right), x_{1}$ is the gear's width $(\mathrm{cm}), x_{2}$ is the number of small gear's teeth, $x_{5}$ and $x_{6}$ are the diameters of two gear shafts, they are integer variables.

$0.001 \leq \phi_{i}(\mathbf{x}) \leq 0.1, \quad$ let $\mu\left(\phi_{i}(\mathbf{x})\right)=20 ; \quad$ if $\quad 0.1 \leq \phi_{i}(\mathbf{x}) \leq 1, \quad$ let $x_{3}$ is the gear's modulus from the first series standard modulus and it's discrete variable, $x_{4}$ is continuous variable which refers to the width of gear housing, with two decimal places. If $\phi_{i}(\mathbf{x})<1$, let $\delta\left(\phi_{i}(\mathbf{x})\right)=0.1$, otherwise let $\delta\left(\phi_{i}(\mathbf{x})\right)=2$. If $\phi_{i}(\mathbf{x})<0.01$, let $\mu\left(\phi_{i}(\mathbf{x})\right)=10$; if

$\mu\left(\phi_{i}(\mathbf{x})\right)=100$, otherwise let $\mu\left(\phi_{i}(\mathbf{x})\right)=300$. The optimum result lists in Table 1. 
Table 1: The solution of reducer optimization

\begin{tabular}{|c|c|c|c|c|c|c|c|}
\hline $\begin{array}{l}\text { Designing } \\
\text { parameters }\end{array}$ & $x_{1}$ & $x_{2}$ & $x_{3}$ & $x_{4}$ & $x_{5}$ & $x_{6}$ & $f(\mathbf{x})$ \\
\hline $\begin{array}{c}\text { Solution } \\
{[14]}\end{array}$ & 13 & 19 & 0.9 & 23.6 & 10 & 13 & 41071 \\
\hline $\begin{array}{c}\text { Solution } \\
\text { [13] }\end{array}$ & 13 & 24 & 0.60 & 23.75 & 10 & 13 & 30675 \\
\hline $\begin{array}{l}\text { Solution } \\
\text { with } \\
\text { ABCOA1.0 }\end{array}$ & 4 & 129 & 0.20 & 14.5 & 10 & 13 & 21814 \\
\hline
\end{tabular}

\section{CONCLUSION}

On the basis of Bee Colony Optimization Algorithm, a new algorithm by introducing constructing dynamic penalty function was presented. Based on Matlab software, the program ABCOA1.0 of Artificial Bee Colony Optimization Algorithm with hybrid discrete variables for the proposed algorithm was developed, the value range of hybrid discrete variables in optimization can be decided rationally by this program. The results show that this algorithm has no special requirements on the characteristics of optimal designing problems, which has a fairly good universal adaptability and a reliable operation of program with a strong ability of overall convergence. These are tremendously significant to advance the efficiency, decrease the weight and reduce the cost.

\section{REFERENCES}

[1] Binwu, D. 1997. Optimization design on automobile gearbox. Journal of Fuzhou University, 25 (5), 59-61.

[2] Hesong, L., Shengmin, C. 2004. Optimum design of automobile gearbox based on MATLAB. Journal of Harbin Institute of Technology, 6 (1), 112114.

[3] Xianglong, D., Weiqiang, Z. 2015. Intensity analysis and structural improvement of electric vehicle battery box based on Workbench. Journal of Mechanical Strength, 37 (2), 312-316.
[4] Zhigang, H., Yang, C., Chaofeng, P. 2016. Multi-objective optimization of Macpherson suspension based on NSGA- II algorithm. Journal of Guangxi University (Nat. Sci Ed), 41(6), 1807-1814.

[5] Xuguang, Z., Chao, J., Sheng, Y. 2015. Multi-objective optimization design of vehicle engine mounting system. Mechanical Science and Technology, 34 (12), 1940-1946.

[6] Chao, W., Youxin, L., Zheming, H. 2007. Optimum design of automobile gearbox based on Lingo10.0 software. Machinery Design and Manufacture $12,75-77$

[7] Wei L.Y., Zhao, M. 2005. A niche hybrid genetic algorithm for global optimization of continuous multimodal function. Applied Mathematics and Computation, 160 (3), 649-661.

[8] Huixin, G., Longting, Z. 2005. Compound genetic algorithm on optimization design of hybrid discrete variables. Journal of Machine Design, 2 2(3), 9-13.

[9] Sheng, W., Xiansheng, G. 2014. Hybrid discrete variable optimization design of planetary gear train based on genetic algorithm. Mechanical Science and Technology, 33 (8), 1150-1154.

[10] Karaboga, D., Akay, B. 2009. A comparative study of Artificial Bee Colony algorithm. Applied Mathematics and Computation, 214 (1), 108132.

[11] Karaboga, D., Basturk, B. 2008. On the performance of artificial bee colony (ABC) algorithm. Applied Soft Computing, 8 (1), 687-697.

[12] Youxin, L., Degang, L., Xiaoyi, C. 2008. Grey robust optimization design of high dimension multi-objective with hybrid discrete variables. Transactions of the Chinese Society of Agricultural, 39 (9), 129-133.

[13] Guo, H., Zhang, L. 2005. Compound genetic algorithm on optimization design of hybrid discrete variables. Journal of Machine Design, 22 (3), 913.

[14] Xu, J. 1996. Mechanical Optimization Design, China Machine Press, Beijing. 